\title{
Aplicações da termografia em saúde: uma revisão de literatura
}

\section{Thermography use in health: A literature review}

\author{
G. C. Mendes*, F. S. Barros** e P. Nohama*** \\ *UTFPR - PPGEB / UniBrasil, Curitiba, Brasil \\ ** UTFPR - PPGEB, Curitiba, Brasil \\ *** UTFPR - PPGEB/CPGEI, Curitiba, Brasil \\ e-mail: gicaroline@yahoo.com.br
}

\begin{abstract}
Resumo: O objetivo da pesquisa foi apresentar uma revisão bibliográfica atualizada a respeito das aplicações da termografia na área da saúde. Foi realizado um levantamento bibliográfico nos meses de novembro e dezembro de 2015, sendo selecionados os artigos publicados entre os anos de 2005 a 2015. A seleção das publicações foi realizada através de leitura criteriosa, correlacionando o conteúdo com o tema a ser pesquisado. Depois de selecionados os artigos, os mesmos foram organizados utilizando como critério o ano de publicação, título, método utilizado, resultados e conclusão. Após a revisão bibliográfica verificou-se que a tecnologia por imagem infravermelha vem evoluindo muito ao longo do tempo. É um método complementar para diagnóstico, com alta acurácia, que vem sendo cada vez mais utilizado na área médica para avaliar alterações fisiológicas e funcionais, sendo de grande importância no diagnóstico complementar e nas avaliações de modalidades terapêuticas a serem empregadas.
\end{abstract}

Palavras-chave: Termografia, Imagem Infravermelha, Dor.

\footnotetext{
Abstract: The purpose of the research was to present an updated bibliographical revision related to the applications of Thermography in health. Within the months of November and December 2015, a bibliographical data collection was performed, and all published articles between 2005 and 2015 were selected. By means of a rigorous reading of such articles, and crossrelating content and subject to be researched. After selecting the articles, they were organized in accordance to their year of publication, title, used method, results and conclusions achieved. Upon bibliographical conclusion, it was concluded that the Infrared Imaging Technology has been substantially evolving throughout time. It stands as a complementary methodology of diagnostics with high accuracy, and which has been more and more used in medicine to assess physiological and functional changes, being of great importance as anadditional diagnostictool, and for the evaluation of therapeutical treatments.
}

Keywords: Thermography, Infrared Imaging, Pain. Introdução

A termografia infravermelha é um método não invasivo, não ionizante, indolor, não prejudicial ao paciente, utilizada para avaliação e medição da temperatura da pele. Baseia-se na radiação infravermelha emitida pela superfície da pele, oferecendo segurança e conforto ao paciente. Esta técnica pode ser utilizada em situações em que a variação de temperatura superficial pode indicar alguma condição atípica, possuindo acurácia quando utilizada para avaliação de dores agudas [1,2].

A inspeção termográfica por infravermelho é realizada utilizando câmeras termográficas. São aparelhos que detectam a energia infravermelha (calor) abrangendo uma faixa de temperatura que vai de $-20^{\circ} \mathrm{C}$ a $1.500^{\circ} \mathrm{C}$, com opção de filtros especiais, fornecendo assim uma imagem de qualidade, independente da presença de sol ou outra fonte de calor intensa. $O$ infravermelho, por ser uma onda abaixo das ondas da cor vermelha, é uma frequência eletromagnética naturalmente emitida por qualquer corpo, com intensidade proporcional à sua temperatura. O sensor da câmara converte a radiação captada em pulsos elétricos, os quais são amplificados e convertidos em sinais digitais. Estes sinais são visualizados como imagens coloridas, em uma escala de cinza ou em cores correspondente a temperatura do objeto. Posteriormente, estas imagens podem ser analisadas por softwares específicos. Devido a sua leveza, câmeras de pequeno tamanho e autonomia podem ser utilizadas em locais de difícil acesso $[3,4,5,6]$.

A termografia infravermelha detecta a luz infravermelha emitida pelo corpo para visualizar mudanças no calor devido a anormalidades no fluxo de sangue da superfície de áreas afetadas. Esta não é uma ferramenta que mostra alterações anatômicas, mas sim um método que mostra alterações fisiológicas. Visualiza sintomas, sendo útil para realizar avaliações e diagnósticos de áreas dolorosas, onde o diagnóstico muitas vezes é baseado em queixas subjetivas [7]. O 
referencial mais importante da termografia é que a distribuição de calor no corpo de um organismo normal é simétrica. Uma câmera de infravermelho é usada para medir a luz infravermelha emitida a partir do corpo e exibe esta na tela e um mapeamento vai sendo realizado. $\mathrm{O}$ comprimento de onda da radiação infravermelha varia entre 0,75 a $100 \mu \mathrm{m}$, não sendo visível aos olhos humanos. Portanto, ao comparar a distribuição de calor do corpo em ambos os lados, a região de interesse é definida e a temperatura média dentro de cada uma destas regiões é calculada para comparar a diferença do lado afetado e não afetado. Um processo doloroso gerado pelos neurotransmissores e maior aumento da circulação local podem ser visualizados por elevação da temperatura local [8].

Para utilizar essa técnica, as salas devem ser climatizadas com temperaturas estabilizadas na faixa de $18,5^{\circ} \mathrm{C}$ a $25^{\circ} \mathrm{C}$ e os pacientes devem ser climatizados por aproximadamente 15 a 30 minutos [9]. Com o passar dos anos, houve um progressivo avanço tecnológico das câmeras e dos softwares. Os sistemas atuais de aquisição de imagem compreendem sofisticadas câmeras térmicas que são acopladas a computadores com programas específicos, sendo as imagens arquivadas de boa qualidade o que torna o método de avaliação mais seguro e preciso $[8,10]$. $\mathrm{O}$ objetivo da pesquisa foi apresentar uma revisão bibliográfica atualizada a respeito das aplicações da termografia na área da saúde.

\section{Materiais e Métodos}

Trata-se de uma pesquisa de revisão bibliográfica, em que foi realizado um levantamento bibliográfico nos meses de novembro e dezembro de 2015, em três bases de dados LILACS, MEDLINE $e$ $S C I E L O$. A busca compreendeu artigos no período de 2005 a 2015, com exceção de duas referências utilizadas para descrever a história da termografia. Como descritores de busca em português foram utilizados: termografia, infravermelho, dor; em inglês: thermography, infrared, pain, todos indexados no DeCS - Descritores em Ciências da Saúde.

Apesar de serem encontrados muitos artigos a respeito da termografia, a seleção das publicações foi realizada através de leitura criteriosa, correlacionando o conteúdo com o tema a ser pesquisado, sendo excluídos os artigos que não apresentavam contextos relacionados ao tema pesquisado.

\section{Resultados e Discussão}

Após a busca realizada nas bases de dados LILACS, MEDLINE e SCIELO foram selecionados 19 artigos que versavam sobre termografia e sua aplicabilidade.

Segundo Cerdeira (2011) [2] a termografia é um instrumento bastante utilizado na construção e engenharia e, apesar de não ser ainda utilizada com muita frequência na área clínica, os estudos demonstram sua sensibilidade, acurácia e importância como uma ferramenta de conclusão diagnóstica.

Segundo Brioschi (2005) [11] quando os resultados de exames anatômicos não são claros ou são contraditórios, a informação adicional fornecida pela termografia pode sugerir um diagnóstico. A imagem infravermelha não demonstra a presença de dor, mas sim as alterações vasomotoras ocorridas em determinadas áreas, como demonstrado no estudo de Bandeira et al(2013) [12] tiveram por objetivo descrever os principais métodos de apoio ao diagnóstico a lesões musculares. Estes citam a termografia como uma importante ferramenta para diagnóstico precoce em lesões musculares, facilitando a decisão dos profissionais da área da saúde para a solicitação e indicação do método, propiciando um diagnóstico mais apropriado.

Luz et al (2010) [13] avaliaram por meio da termografia, a adaptação de uma prótese híbrida de extremidade superior, de um indivíduo do sexo masculino, amputado proximal de braço esquerdo. Após $8 \mathrm{~h}$ de uso ininterrupto, a prótese foi retirada e então realizaram a primeira imagem. Após 20 minutos de repouso, quando já havia alcançado o equilíbrio térmico, realizaram a segunda imagem. Após análise das imagens, constataram elevadas temperaturas na região de tórax e axila contralateral ao coto, por onde passa o tirante de fixação da prótese, coincidindo com a queixa de desconforto do indivíduo, indicando atrito. Observaram também maior temperatura no ombro anterior homolateral à amputação o que caracterizou sobrecarga, e por fim, a baixa temperatura no segmento residual à amputação indicou pouca circulação demonstrando dificuldade de adaptação (Figura 1).

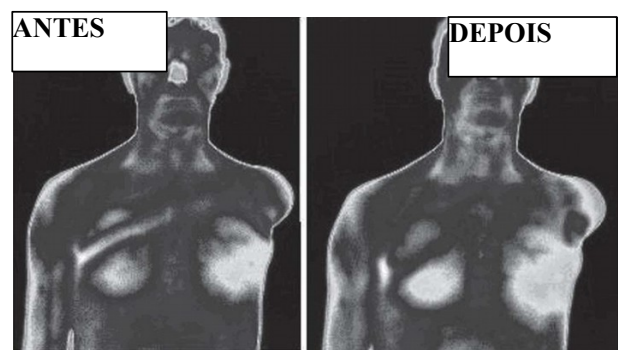

Figura 1 - Região do tórax ao longo do tirante de fixação da prótese, antes e depois do equilíbrio térmico: temperatura mais elevada antes e permanência de alta temperatura depois, na região da axila homolateral ao membro íntegro, coincidindo com a região relatada de maior desconforto. Fonte: Luz et.al., 2010.

O estudo de Luz et al (2010) [13] onde afirmam que a baixa temperatura no segmento residual à amputação indicou pouca circulação, corrobora com o achado de Brito et al (2015) [14]. Estes realizaram um estudo com 33 indivíduos com dor crônica lombar, que seriam avaliados pré e pós-intervenção fisioterapêutica com uso da termografia (Figura 2) e da EVA (escala visual analógica). Os tratamentos não minimizaram a 
temperatura da região lombar, mas o nível de dor segundo a EVA diminuiu significativamente. Pode ser justificado pelo estudo de Ring e Ammer (2012) [15] que afirmam que em lesões crônicas pode ser encontrada diminuição da temperatura, causada por uma contração muscular reduzida e diminuição do fluxo sanguíneo local.

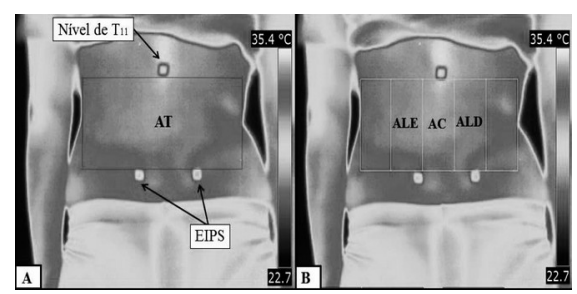

Figura 2 - Delimitações para análise das imagens termográficas: AT = área total (A); ALE = área lombar esquerda; $\mathrm{AC}$ = área central; e ALD = área lombar direita (B). Fonte: Brito et.al., 2015.

Bandeira, et al (2012) [16] verificaram em sua pesquisa, a importância de controlar a camada de gordura da área avaliada, pois a mesma interfere nos valores absolutos de temperatura, podendo influenciar de maneira significativa nos resultados. $\mathrm{O}$ diagnóstico de anormalidades neurológicas e musculoesqueléticas por termografia infravermelha é baseado na assimetria de cores entre os lados normal e sintomático, mudança no gradiente de radiação normal dos membros ou entre os lados medial e lateral ou um distúrbio no padrão normal fisiológico de distribuição da radiação.

Nogueira (2010) [17] em seu estudo propôs uma avaliação das polpas digitais das mãos de grávidas normotensas e grávidas hipertensas, para observar se haveria diferença na temperatura local. A amostra foi composta por 81 gestantes, no período entre a $20^{\mathrm{a}}$ e $38^{\mathrm{a}}$ semana de gestação, sendo que destas 50 eram normotensas e 31 hipertensas. Como resultado, pode-se observar na Figura 3A, paciente normotensa, uma hiper radiação (área branca) nas polpas digitais e na palma da mão, diferentemente do demonstrado na Figura 3B, paciente hipertensa, onde a hiper radiação está presente somente na palma da mão.

$\mathrm{O}$ aumento de temperatura das polpas digitais reflete no aumento do fluxo sanguíneo periférico. A vasoconstrição cutânea, presente nos quadros hipertensivos, prejudica a termorregulação, impedindo a troca de calor com o ambiente, o que pode interferir na pressão arterial. A média de temperatura das polpas digitais das mãos das grávidas normotensas e hipertensas teve uma diferença significativa de $1,28^{\circ} \mathrm{C}$, sendo que as grávidas hipertensas $\left(34,96^{\circ} \mathrm{C}\right)$ tem maiores chances de terem a polpa digital das mãos mais quentes que as grávidas normotensas $\left(33,96^{\circ} \mathrm{C}\right)$.

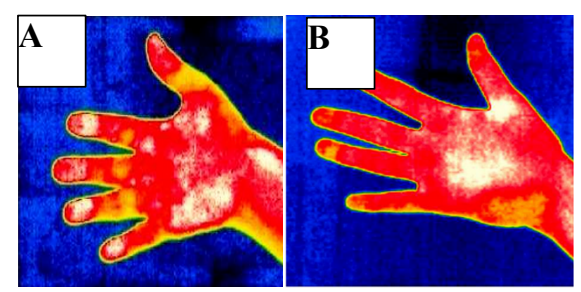

Figura 3 - Na figura (A) observa-se hiper radiação (áreas brancas) nas polpas digitais e na palma da mão. Na figura (B) observa-se hiper radiação na palma da mão. Fonte: Nogueira, 2010.

Embora a imagem térmica ainda não seja amplamente utilizada na prática clínica para avaliação de sinais da doença do pé diabético, NETTEN et al. (2013) [18] realizaram um estudo com 15 indivíduos, divididos em três subgrupos. Os grupos foram divididos em indivíduos sem complicações do pé diabético, com complicações locais e com complicações difusas. Nos indivíduos sem complicações houve uma pequena diferença de temperatura entre os pés. Os indivíduos com complicações locais, como úlceras não infectadas e não isquêmicas e calos abundantes, mostrou aumento da temperatura local em $2^{\circ} \mathrm{C}$ comparando com o membro contralateral. Nos indivíduos com complicações difusas, como úlceras com osteomielite, pé de Charcot, obtiveram resultados de temperatura maior que $3^{\circ} \mathrm{C}$ em comparação ao membro contralateral (Figura 4).

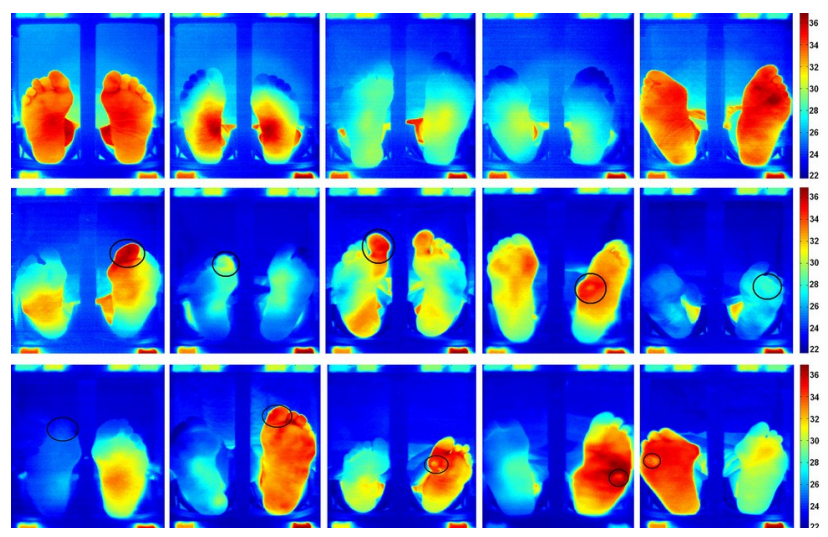

Figura 4 - Na linha superior, da esquerda para a direita, pacientes de 1 a 5 , imagens de cinco pacientes sem complicações nos pés; linha do meio, esquerda para a direita, os pacientes de 6 a 10, com complicações locais nos pés e nas imagens de 11 a 15, linha de baixo, da esquerda para a direita, pacientes com complicações difusas nos pés. Fonte: Netten et al., 2013.

Assim como Netten et al (2013) [18], Brioschi et al (2007) [19] descreveram em seu estudo que o objetivo da termografia infravermelha no pé diabético seria a de detectar precocemente as alterações de sensibilidade dos pés, déficits circulatórios, lesões inflamatórias e infecciosas, sobrecarga mecânica. Na Figura 5, observa-se o sinal de "amputação em bota", onde delimita-se exatamente o nível de comprometimento microvascular, realizando assim amputações mais seguras e precisas, evitando reamputações. Relatam também a relação da 
temperatura plantar e o grau de acometimento do pé diabético (Figura 6), sendo que quando houver diferença térmica maior que $2,2^{\circ} \mathrm{C}$ entre os lados direito e esquerdo, o indivíduo dever ser avaliado também por uma equipe médica.

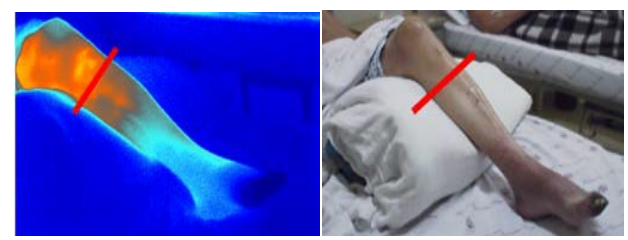

Figura 5 - Termometria infravermelha delimitando nível de amputação em paciente diabético com isquemia de membro inferior esquerdo. Fonte: Brioschi et al., 2007.

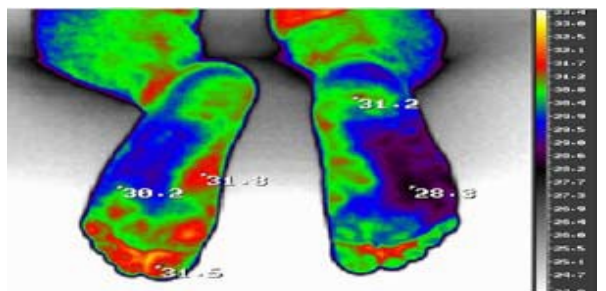

Figura 6 - Pé esquerdo com neuropatia diabética, presença de múltiplos focos hiper radiantes (hot spots) em região plantar do ante pé com risco de ulceração. Fonte: Brioschi et al., 2007.

\section{Conclusão}

Após a revisão bibliográfica, verificou-se que a tecnologia por imagem infravermelha vem evoluindo muito ao longo do tempo. É um método complementar para diagnóstico, com alta acurácia, que vem sendo cada vez mais utilizado na área médica para avaliar alterações fisiológicas e funcionais, sendo de grande importância no diagnóstico complementar e nas avaliações de modalidades terapêuticas a serem empregadas. Esta tecnologia pode ser de grande eficácia para avaliação de pontos dolorosos, lesões musculares, adaptações de próteses e doenças vasculares. Pode-se observar que a avaliação termográfica auxilia também no diagnóstico precoce do pé diabético, o que poderia contribuir para a prevenção de maiores complicações. Sugerem-se novos estudos quanto ao uso da termografia em indivíduos com hipertensão arterial sistêmica.

\section{Referências}

[1] Dibai-Filho AV e Guirro RRJ. Evaluation of Myofascial Trigger Points Using Infrared Thermography: A Critical Review of the Literature. Journal of Manipulative and Physiological Therapeutics. 2014; 38 (1).

[2] Cerdeira F, Vásquez ME, Collazo J e Granada E. Applicability of infrared thermography to the study of the behavior of stone panels as building envelopes. Energy and Buildings, Oxford. 2011; v. 43: 1845- 1851.

[3] Louvain LC, Cabral LG e Gomes KR. Aplicação da Termografia na Manutenção Preditiva. Bolsista de
Valor:Revista de divulgação do Projeto Universidade Petrobras e IF Fluminense, 2010, (1): 109-112.

[4] Mendonça LV. Termografia por Infravermelhos Inspeção de Betão. Engenharia \& Vida, Lisboa, Setembro 2005; 16 (1): 53-57.

[5] Sanches IJ. Superposição de imagens de termografia e ressonância magnética: Uma nova modalidade de imagem médica tridimensional. 2009. 168 f. Tese (Doutorado em Engenharia Biomédica) Universidade Tecnológica Federal do Paraná, Curitiba, 2009.

[6] Altoé L e Oliveira Filho D. Termografia infravermelha aplicada à inspeção de edifícios. Acta Tecnológica, 2012; 1 (7): 55-59.

[7] Nahm FS. Infrared Thermography in Pain Medicine. The Korean Journal of Pain. Julho 2013, 26 (3): 219-22.

[8] Trotta J e Ulbricht L. Termografia no Diagnóstico Complementar de Doenças Músculo Esqueléticas. Pan American Journal of Medical Thermology. 2015; 2 (1): 7-13.

[9] Arfaoui A, Bouzid MA, Pron H, Taiar R e Polidori G. Application of Infrared Thermography as a Diagnostic Tool of Knee Osteoarthritis. Journal Of Thermal Science and Technology. 2012; 7 (1): 227-235.

[10] Meira LF, Krueger E, Neves EB, Nohama P. E Souza MA. Termografia na Área Biomédica. Pan American Journal of Medical Thermology. 2014; 1 (1): 31-41

[11] Brioschi, M. L. Value Of Infrared Imaging In Pain Evaluation. Rev. Dor 2005 - Jan./Mar., 6 (1): 514-524.

[12] Bandeira F, Neves B, Barroso GC e Nohama P. Métodos de apoio ao diagnóstico de lesões musculares. Revista Brasileira de Inovação Tecnológica em Saúde, online, 2013, p. 27-44.

[13] Luz SCT, Oliveira TP, Andrade MC, Ávila $\mathrm{AOV}$, Rosa FJB. Adaptação à prótese híbrida de extremidade superior: estudo termográfico de um caso. Fisioterapia em Pesquisa. 2010; 17 (2): 173-7.

[14] Brito JD, Schimit EFD, Nóbrega SR, AraújoNeto AS, Ferreira JJA, Andrade PR e Santos HH. Alterações termográficas na lombalgia crônica sob tratamento fisioterapêutico: ensaio clínico controlado e randomizado. ConScientice Saúde, 2015; 14 (1): 89-98.

[15] Ring EFJ, Ammer K. Infrared thermal imaging in medicine. Physiol Meas. 2012; 33 (3): 3346.

[16] Bandeira F, Moura MAM, Souza MA, Nohama $\mathrm{P}$ e Neves EB. Pode a termografia auxiliar no diagnóstico de lesões musculares em atletas de futebol? Revista Brasileira de Medicina Esporte, Jul./Ago., 2012,18 (4): 246-251.

[17] Nogueira FE. Dissertação de Mestrado: Análise comparative das temperaturas das polpas digitais entre gestantes hipertensas e 
normotensas usando termografia de infravermelho, Fortaleza, 2010.

[18] Netten JJV, Baal JGV, Liu C, Heijden FVD e Bus AS. Infrared Thermal Imaging for Automated Detection of Diabetic Foot Complications. Journal of Diabetes Science and Technology. Set. 2013; vol.7: 1122-1129.

[19] Brioschi ML, Mehl A, Oliveira AGN, Freitas MAS, Macedo JF, Matias JEF, Macedo RAC. Exame de termometria cutânea infravermelha na avaliação do pé diabético. Rev. Med. Paraná, Curitiba, 2007; 65 (1): 33-41. 